\title{
In memoriam Raymond Battegay
}

Im Alter von 89 Jahren ist Raymond Battegay am 4. Oktober 2016 gestorben. Wir verlieren damit einen Kollegen und Freund, der die Geschichte der Psychiatrie und Psychotherapie nicht nur in der Schweiz, sondern weltweit massgeblich geprägt hat.

Raymond Battegay war zutiefst geprägt durch sein jüdisches Selbstverständnis und war verbunden mit der jüdischen Geschichte und Religion. Er ist in Biel aufgewachsen, hat die Schulen in Basel besucht, an der Universität Basel Medizin studiert und 1952 das eidgenössische Arztdiplom erlangt. Nach einer Assistenzzeit an der Psychiatrischen Universitätsklinik Basel arbeitete er ein Jahr, 1954/55, am Beilinson-Spital in Petah Tiqva bei Tel Aviv, bevor er 1955 an die Psychiatrische Universitätsklinik Basel zurückkehrte. In Israel lernte er seine Frau Shulamit, geborene Fitaya, aus einer berühmten Rabbinerfamilie in Bagdad stammend, kennen und auf den ersten Blick lieben. Vor kurzem konnten Raymond und Shulamit glücklich das 60-Jahr-Ehejubiläum feiern.

\section{Mit unzähligen Veröffentlichungen und Lehr- büchern erlangte Raymond Battegay in der ganzen Welt als Wissenschaftler und Psychiater grosse Anerkennung und Ehre.}

Raymond Battegay war ein grossartiger Mensch, ein wahrer Humanist und ein begnadeter Arzt, der sich unablässig, unermüdlich, konsequent, kompromisslos, mit einer immensen Kraft und einem grossen Engagement für das Leiden der Menschen, für Gerechtigkeit, für Toleranz und das Miteinander der Religionen einsetzte. So genoss und schätze er den Diskurs und das Miteinander mit Kolleginnen, Kollegen und Freunden, weit über die Psychiatrie hinaus und zu anderen Berufsgruppen.

1958 wurde Raymond Battegay an der Psychiatrischen Universitätsklinik Basel zum Oberarzt ernannt. 1969 wurde er Extraordinarius und 1976 Ordinarius ad personam der Universität Basel sowie Chefarzt der Psychiatrischen Universitätspoliklinik Basel, die er über 30 Jahre bis zu seiner Emeritierung 1997 leitete. Von 1985-1987 war er Dekan der Medizinischen Fakultät der Universität Basel. Er hat hier massgeblich eine moderne, v.a. ambulante Psychiatrie aufgebaut und mitgestaltet.

Mit unzähligen Veröffentlichungen und Lehrbüchern über Themen quer durch die Psychiatrie, von der Neurosenlehre und Gruppentherapie bis hin zur Psycho-

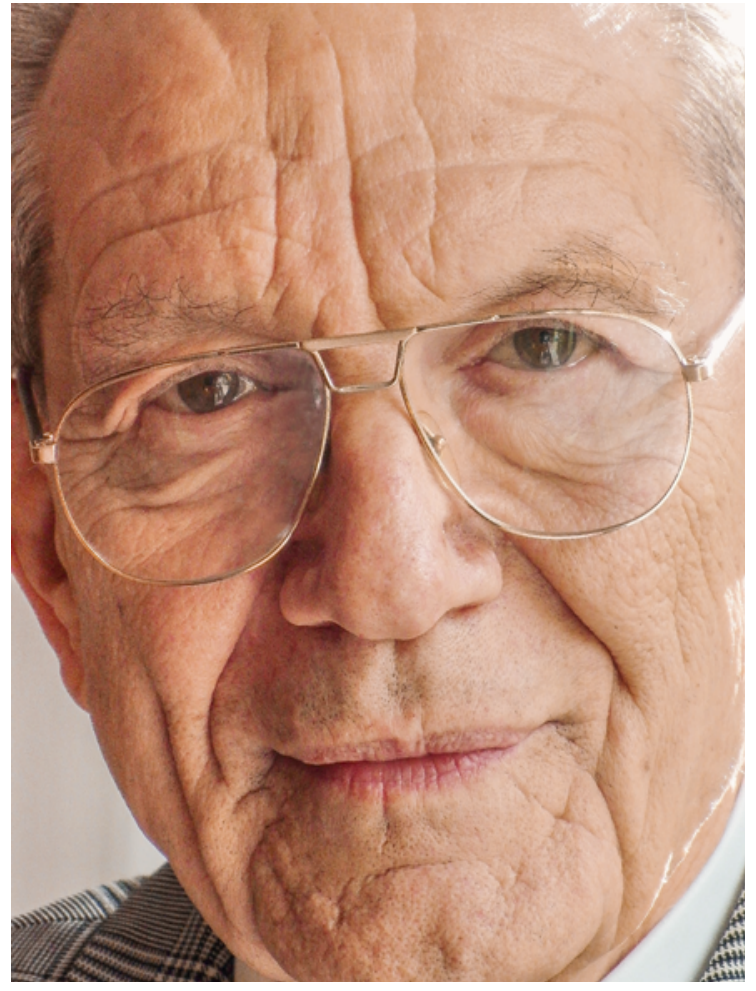

Professor Dr. Raymond Battegay (27.6.1927-4.10.2016).

pharmakologie und Sozialpsychiatrie, erlangte Raymond Battegay nicht nur schweizweit, sondern in der ganzen Welt als Wissenschaftler und Psychiater grosse Anerkennung und Ehre. Er war Mitbegründer der Gruppentherapie und aufgrund seiner Eloquenz ein begehrter Referent an unzähligen Kongressen weltweit. Als Wissenschaftler widmete sich Raymond Battegay in einer Pionierzeit der Psychiatrie in den 50er und 60er Jahren der Erforschung und Optimierung der pharmakologischen Behandlung von Menschen, die

\section{Raymond Battegay betreute und behandelte} unendlich viele Patienten ungeachtet ihrer Herkunft, ihres Alters, der Religion oder des sozialen Status.

an einer Schizophrenie und an Depressionen litten. Seine Hauptforschung betraf die Weiterentwicklung der aus den USA kommenden Gruppenpsychotherapie in Europa sowie des Verhaltens von kranken und gesunden Menschen in der Gruppe. Hier führte er neue Konzepte ein, die für die fokussierte Kurzpsychotherapie wesentlich waren. 
Ein hervorstehendes Merkmal von Raymond Battegay ist, dass er verschiedene Strömungen integrierte noch lange vor einer Zeit, da über die Implementierung von Forschung und Interdisziplinarität gesprochen wurde. Sein Anliegen war stets, die Situation für die Patienten zu verbessern. Das wissenschaftliche Euvre von Raymond Battegay umfasst mehr als 700 Arbeiten und über 25 Bücher als Autor, Mitautor bzw. Mitherausgeber über die verschiedensten psychiatrischen und psychotherapeutischen Themen. Raymond Battegay war in zahlreichen Gremien Mitglied, unter anderem war er Mitinitiant der Seniorenuniversität Basel, zehn Jahre Mitglied der Regenz der Universität Basel und Mitglied in vielen schweizerischen und internationalen psychiatrischen Gremien.

\section{Humor, Selbstironie, Witz gemischt mit theatralem Geschick gehörten zum Wesen von Raymond Battegay.}

In der Basler Psychiatrischen Universitätspoliklinik leitete Raymond Battegay parallel zu seinen anderen Aufgaben und Verpflichtungen regelmässig verschiedene eigene Gruppen. In den 70er Jahren gründete er eine Psychotherapiegruppe für schizophrene $\mathrm{Pa}$ tienten und eine für deren Angehörige. Damals galt in der psychiatrischen Fachwelt die Meinung, dass schizophrene Patienten weder einzeln, geschweige denn in einer Gruppe psychotherapeutisch, schon gar nicht psychoanalytisch behandelbar wären. Raymond Battegay bewies mit dieser Gruppe, vielen Publikationen und seinem Buch Der Mensch in der Gruppe das Gegenteil und erntete weltweit grosse Anerkennung, fand viele Mitstreiter und Nachfolger. Diese Gruppe fand sich über 40 Jahre lang regelmässig zweiwöchentlich zusammen, war damit weltweit wohl eine der am längsten geführte Gruppentherapien, und es entstand eine tiefe Bezogenheit und eine berührende Freundschaft zwischen allen Mitgliedern. Für Raymond Battegay war die Gruppe ein Mikrokosmos, in dem sich die Welt spiegelte. Er gestaltete eine Atmosphäre, in der es den anwesenden Patienten gelang, sich zu äussern, für sich einzustehen, zuzuhören, emotional sich zu beteiligen, Konflikte auszutragen (und es gab deren viele), eigene und anderer Freuden und Leiden zu teilen, zu partizipieren, sich und die anderen zu akzeptieren.

Raymond Battegay blieb, neben all seinen Verpflichtungen, Ämtern und Aufgaben, stets als Arzt tätig, der unendlich viele Patienten ungeachtet ihrer Herkunft, ihres Alters, der Religion oder des sozialen Status betreute und behandelte, sich für diese und für deren Angehörige und Familien vollends und leidenschaftlich einsetzte. Wenn das Leiden der Menschen nicht nur durch Krankheit, sondern auch durch Gesetzgebungen, politische Entscheide, Kriege, Flucht, Diskriminierungen oder ihre Religionszugehörigkeit entstand, lehnte sich Raymond Battegay mit lauter Stimme und grosser Zivilcourage, oft gegen den Strom der Zeit schwimmend, dagegen auf, gab den Betroffenen in der Öffentlichkeit durch seine Kolumnen in der hiesigen Zeitung eine Stimme. Diese Kolumnen wühlten auf, wurden von Bürgern und Politikern diskutiert und kommentiert.

Seit der Emeritierung 1997 arbeitete Raymond Battegay in unserer Praxisgemeinschaft. In der Folge und bis zum vergangenen Jahr verbrachten wir über 18 Jahre lang mit viel Freude zusammen in unserer Gemeinschaftspraxis an der Delsbergerallee in Basel, arbeiteten Tür an Tür. Die Praxisatmosphäre war geprägt von viel Fröhlichkeit, es wurde viel gelacht und gescherzt, so dass einige Patienten, die neu zu uns kamen, sich fragten, ob sie an der richtigen Adresse seien. Humor, Selbstironie, Witz gemischt mit theatralem Geschick gehörten zum Wesen von Raymond Battegay.

Wir haben mit Raymond Battegay nicht nur einen höchst kreativen Wissenschaftler und Arzt verloren, sondern auch einen grossartigen Menschen, der sich bis zum Ende seines Lebens mit ungeheurem Engagement für psychisch leidende Menschen eingesetzt hat. Wir sind ihm zu grossem Dank verpflichtet.

Dr. Maurizio Molinari und Prof. Dr. Udo Rauchfleisch, Basel 